\title{
Three-Phase AC Linear Proportional Solenoid Actuator with Zero Hysteresis in Current-Thrust Force Characteristics
}

\author{
Yuta Tominari ${ }^{1}$, and Yasukazu Sato $^{2}$, Member, IEEE \\ ${ }^{1}$ Graduate School of Engineering, Yokohama National University, Yokohama, Kanagawa 240-8501, Japan \\ ${ }^{2}$ Department of Mechanical Engineering, Yokohama National University, Yokohama, Kanagawa 240-8501, Japan
}

\begin{abstract}
An electromagnetic proportional solenoid is used as a force control device to generate thrust force acting on a valve element in a hydraulic pressure control valve. However, a conventional proportional solenoid has the problem of hysteresis appearing in its currentthrust force characteristics, which is induced by friction and magnetic hysteresis. In order to reduce hysteresis, this paper proposes the three-phase AC solenoid that reproduces a pseudo-magnetic bearing. Its prototype reduces hysteresis and achieves the same performance as the conventional proportional solenoid. The magnetic pole shape and the current input condition considering the magnetic flux density saturation are presented based on electromagnetic field analysis.
\end{abstract}

Index Terms-Actuator, Linear Solenoid, Hysteresis, Thrust Force Control.

\section{INTRODUCTION}

A solenoid actuator shown in Fig. 1 is widely used in electromechanical system as a short stroke linear motor producing an electromagnetic thrust force according to the coil DC current. For a general solenoid actuator, the thrust force increases in proportion to the square of the reciprocal of the air gap between the armature and stator under the constant coil DC current [1], [2]. Because of its force-current characteristics, the armature position should be limited just on the maximum or the minimum airgap, as it cannot control the position continuously when in between. Compared with the general solenoid actuator, a proportional solenoid shown in Fig. 2 can produce the thrust force in proportion to the coil DC current insensitive to the armature position. Therefore, the proportional solenoid plays a role as a current-thrust force converter to operate a valve in oil hydraulic or pneumatic system [3]. In the proportional solenoid, the magnetic flux is controlled by the special shape magnetic pole in its magnetic circuit, with a combination of magnetic saturation and magnetic flux leakage. The proportional solenoid can produce the thrust force proportional to the DC current irrespective of the position of the armature within a certain distance (control zone).

Though the proportional solenoid theoretically has linear thrust force-current characteristics by the thrust force production principle, an actual proportional solenoid has hysteresis, as shown in Fig. 3. These characteristics were obtained by measurement of a conventional proportional solenoid, as an example. The simulation results based on electromagnetic field analysis without the friction and the magnetic hysteresis are shown along with the measurement results. There are two possible causes of hysteresis. One cause is the mechanical friction due to eccentricity of the armature

Manuscript received November 5, 2018; revised December 10, 2018; accepted July 1, 2015. Date of publication July 10, 2015; date of current version July 31, 2015. Corresponding author: Yasukazu Sato (e-mail: satoyasukazu-zm@ynu.ac.jp). Yasukazu Sato and Yuta Tominari contributed equally.

Color versions of one or more of the figures in this paper are available online at http://ieeexplore.ieee.org.

Digital Object Identifier (inserted by IEEE).
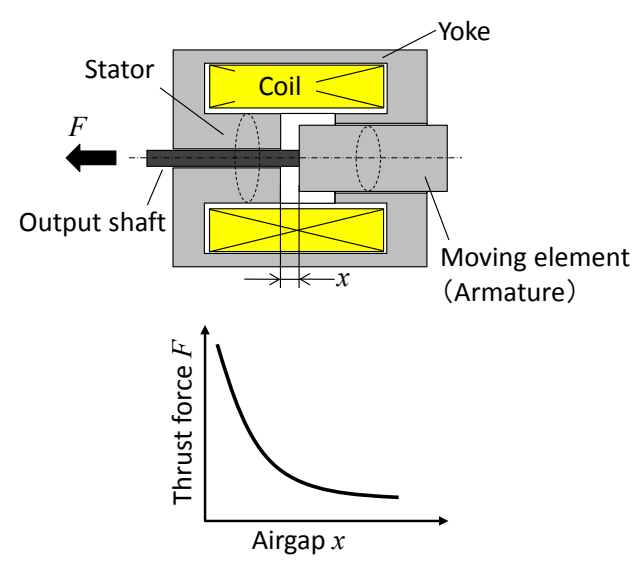

Fig. 1. Plunger type solenoid for two-position control

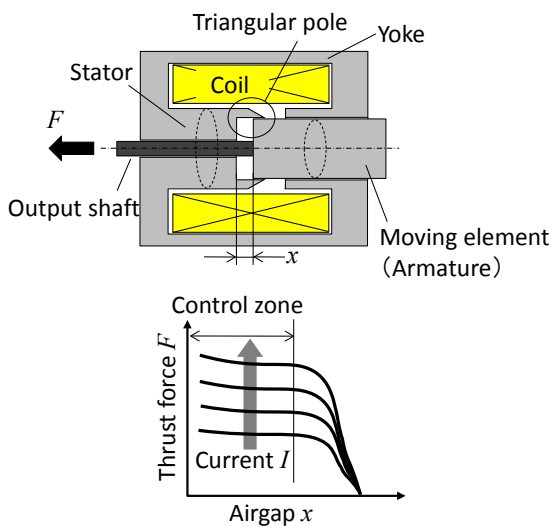

Fig. 2. Proportional solenoid for continuous thrust force control

in the sleeve inside the solenoid, as shown in Fig. 4. The other is the magnetic hysteresis in the magnetic circuit of the proportional solenoid. The solenoid has a certain radial clearance for armature movement. At a part of circumference 


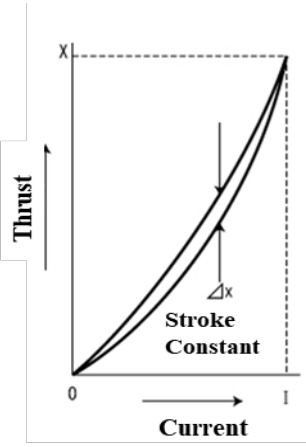

(a) Thrust force-Current

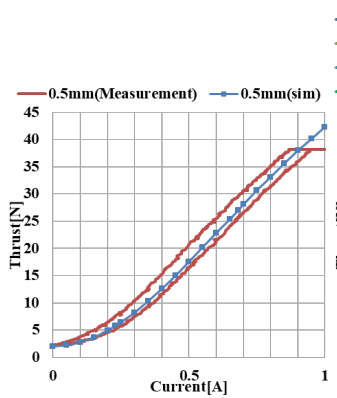

(c) Example of thrust force - current measurement with simulation

Fig. 3. Hysteresis of the thrust force-current characteristics in the fixed armature position, the thrust force-stroke characteristics under the constant coil DC current, in a conventional proportional solenoid ( $\Delta x$ : width of hysteresis)

of armature, the smaller the radial clearance due to eccentricity, the larger the electromagnetic attractive force becomes between the yoke and the cylindrical surface of the armature drastically. In the condition where the armature touches to sleeve, the mechanical friction at the contact area induces the hysteresis. To avoid the eccentricity of the armature in a conventional proportional solenoid, mechanical linear bearings are incorporated to keep the armature axis at the center of the sleeve. Installation of the linear bearings makes the proportional solenoid structure more complicated. In order to reduce the hysteresis caused by the static mechanical friction and magnetic hysteresis, the dither, which is a high frequency and small amplitude AC current, is added on the coil DC current [4], [5]. However, the dither induces a slight fluctuation in the thrust force [6]-[8].

In this paper, we aim to reduce the hysteresis on the thrust force -current characteristics of the proportional solenoid due to the mechanical friction, and to avoid the fluctuation of the thrust force due to the dither. We propose a proportional solenoid driven by a three-phase AC current, abbreviated as TPAC-PS (Three-Phase AC Proportional Solenoid), and present that it can reduce the hysteresis, and can achieve the same performance as the conventional proportional solenoid driven by the coil DC current with dither.

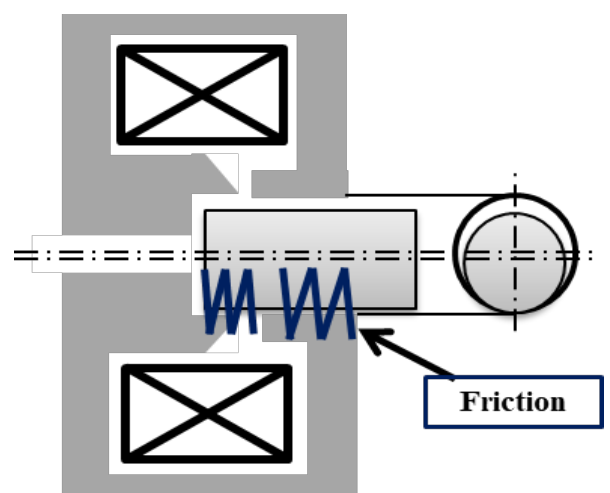

Fig. 4. Mechanical friction due to eccentricity of armature in a conventional proportional solenoid

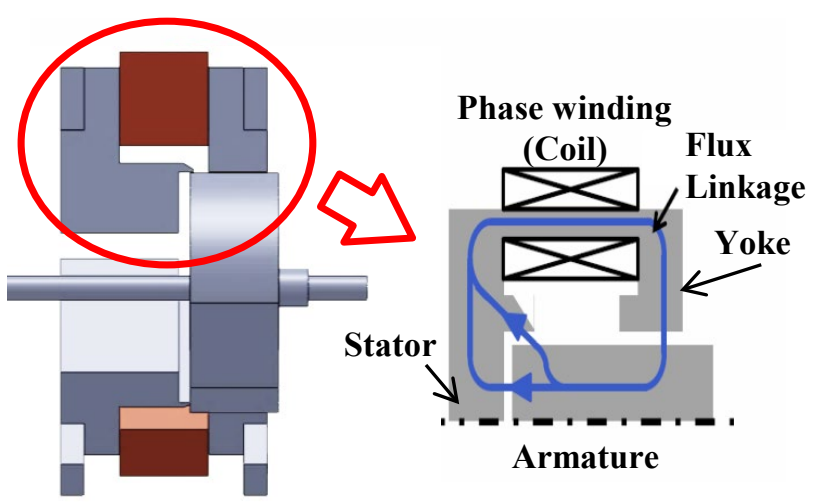

Fig. 5. Three-phase AC proportional solenoid (TPAC-PS)

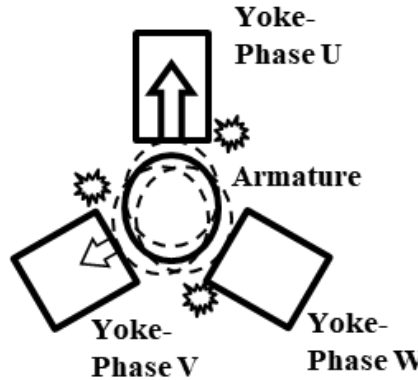

(a) Low frequency three-phase AC (Touch down occurs)

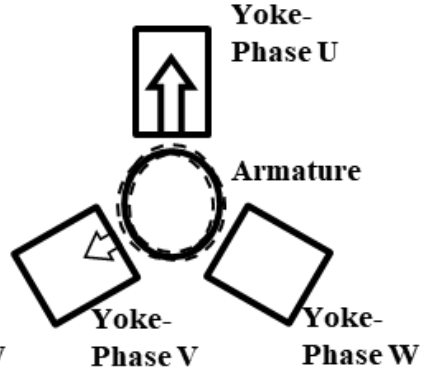

(b) High frequency three-phase AC (Magnetic bearing)
Fig. 6. Lateral force generated by three-phase $\mathrm{AC}$ energization in each yoke in TPAC-PC

\section{THEORY OF THREE-PHASE AC PROPORTIONAL SOLENOID, TPAC-PS}

\section{A. Structure of TPAC-PS}

In order to reduce the hysteresis appearing on a conventional proportional solenoid driven by the coil DC current, this study proposes a proportional solenoid driven by the coil current of 
three-phase AC. Figure 5 shows the three-phase AC proportional solenoid, having a structure that can form pseudomagnetic bearings that avoid continuous touch of the armature to the sleeve. The yokes are around and orthogonal to the moving direction of the armature to conduct its magnetic flux. The arrangement of the yoke, for each phase of AC excitation of the windings, prevents the contact between the armature and the sleeve, and reduces the influence of mechanical friction. As shown in Fig. 6, for low frequency three-phase AC, the lateral attractive force makes the armature touchdown to the yoke and sleeve, then noise and vibration, and friction are generated. For a high frequency three-phase $\mathrm{AC}$, the direction of the lateral attractive force always switches before the armature touchdown to the yoke and behaves just like a magnetic bearing. Because the armature keeps some radial clearance to all yokes, the hysteresis caused by the mechanical friction is remarkably reduced.

\section{B. Thrust force of TPAC-PS}

Regarding the thrust force of TPAC-PS, the attraction force $F$ [N] of the electromagnet can be expressed by Eq. (1) [2]

$$
F=\frac{1}{2} U^{2} \frac{d P}{d x}
$$

where $P, U$, and $x$ are the permeance [ $\mathrm{Wb} / \mathrm{AT}]$, magnetomotive force $\left[\mathrm{A}^{2}\right]$, and airgap $[\mathrm{m}]$, respectively. In the case where two ferromagnetic pole faces of the cross sectional area $S\left[\mathrm{~m}^{2}\right]$ are oriented parallel to each other with a airgap $x[\mathrm{~m}]$,

$$
\frac{d P}{d x}=-\frac{\mu_{0} S}{x^{2}}
$$

is obtained because the permeance of the airgap is $P=\mu_{0} S / x$, with the permeability of air $\mu_{0}$. According to Eqs. (1) and (2), the force $F$ is expressed by Eq. (3)

$$
F=-\frac{1}{2} U^{2} \frac{\mu_{0} S}{x^{2}}=-\frac{1}{2} U^{2} \frac{B_{g}^{2} S}{\mu_{0}}=-\frac{1}{2} \frac{\Phi^{2}}{\mu_{0} S}
$$

where the negative sign indicates that the force is an attractive force. $B_{g}\left[\mathrm{~Wb} / \mathrm{m}^{2}\right]$ is the magnetic flux density, and $\Phi[\mathrm{Wb}]$ is the magnetic flux at the airgap.

The magnetic circuit of the TPAC-PS can be represented using the electric circuit analogy, as shown in Fig. 7. In the circuit, $R, U$, and $\Phi$ represent the reluctance, magnetomotive force, and magnetic flux, respectively.

The thrust force $F$ of the TPAC-PS is the sum of the attractive forces $F_{U}, F_{V}$, and $F_{W}[\mathrm{~N}]$ by the magnetic flux at the airgap between the armature and the stator of each phase magnetic pole. Therefore, it becomes as shown in Eqs. (4) and (5)

$$
\begin{array}{r}
F=F_{U}+F_{V}+F_{W} \\
=\frac{1}{2 \mu_{0} S}\left(\Phi_{U}^{2}+\Phi_{V}^{2}+\Phi_{W}{ }^{2}\right),
\end{array}
$$

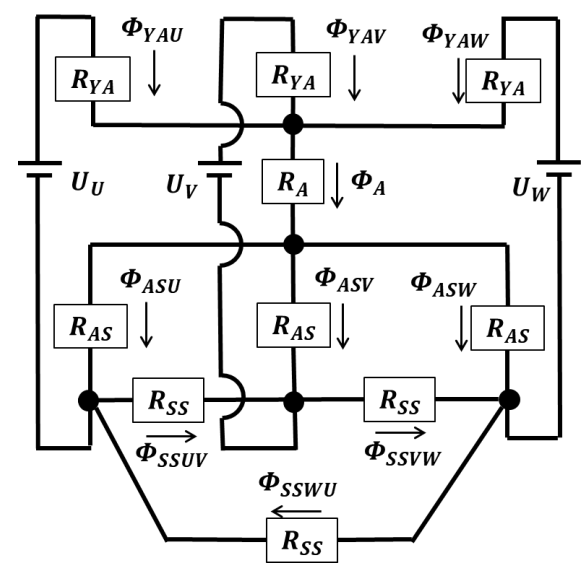

$R_{A S}$ : Reluctance of armature-stator gap [AT / Wb]

$R_{Y A}:$ Reluctance of yoke-armature gap [AT / Wb]

$R_{S S}$ : Reluctance of stator-stator gap [AT / Wb]

$R_{A}$ : Reluctance of armature [AT / Wb]

$U_{U}$ : Magnetomotive force by $\mathrm{U}$ phase current [AT]

$U_{V}$ : Magnetomotive force by $\mathrm{V}$ phase current [AT]

$U_{W}$ : Magnetomotive force by $\mathrm{W}$ phase current [AT]

$\Phi_{A S U, V, W}$ : Magnetic flux in $R_{A S}$ at $\mathrm{U}, \mathrm{V}, \mathrm{W}$ magnetic poles [Wb]

$\Phi_{Y A U, V, W}$ : Magnetic flux in $R_{Y A}$ at $\mathrm{U}, \mathrm{V}, \mathrm{W}$ magnetic poles [Wb]

$\Phi_{S S U V, V W, W U}$ : Magnetic flux in $R_{S S}$ at $\mathrm{U}-\mathrm{V}, \mathrm{V}-\mathrm{W}, \mathrm{W}-\mathrm{U}$ magnetic poles [Wb]

$\Phi_{A}$ : Magnetic flux in $R_{A S}[\mathrm{~Wb}]$

Fig. 7. Magnetic circuit of TPAC-PS

$$
\begin{aligned}
F= & \frac{1}{2 \mu_{0} S} C 1_{x}{ }^{2}\left\{C 2_{x}\left(U_{U}{ }^{2}+U_{V}{ }^{2}+U_{W}{ }^{2}\right)\right. \\
& \left.+C 3_{x}\left(U_{U} U_{V}+U_{V} U_{W}+U_{W} U_{U}\right)\right\}
\end{aligned}
$$

where $C 1_{x}, C 2_{x}$, and $C 3_{x}$ are the constants by reciprocal of armature-stator airgap. Regarding the magnetomotive force $U_{U}$, $U_{V}$, and $U_{W}$, three-phase alternating current energizes each coil of the number of turns $N$ [-], expressed by the combination of the offset DC $I_{D C}[\mathrm{~A}]$ and the amplitude $I_{A C}[\mathrm{~A}]$ of the threephase AC shifted by $2 \pi / 3$ from each other as follows:

$$
\left\{\begin{array}{l}
U_{U}=N\left\{I_{A C} \sin (\omega t)+I_{D C}\right\} \\
U_{V}=N\left\{I_{A C} \sin (\omega t+2 \pi / 3)+I_{D C}\right\} \\
U_{W}=N\left\{I_{A C} \sin (\omega t-2 \pi / 3)+I_{D C}\right\}
\end{array}\right.
$$

Substituting these into Eq. (5) results to Eq. (7):

$$
\begin{aligned}
F= & \frac{1}{2 \mu_{0} S} C 1_{x}^{2}\left\{C 2_{x}\left(\frac{3}{2} I_{A C}^{2}+3 I_{D C}{ }^{2}\right)\right. \\
& \left.+C 3_{x}\left(-\frac{3}{4} I_{A C}{ }^{2}+3 I_{D C}{ }^{2}\right)\right\}
\end{aligned}
$$

This means that the fluctuation by $\mathrm{AC}$ component does not 


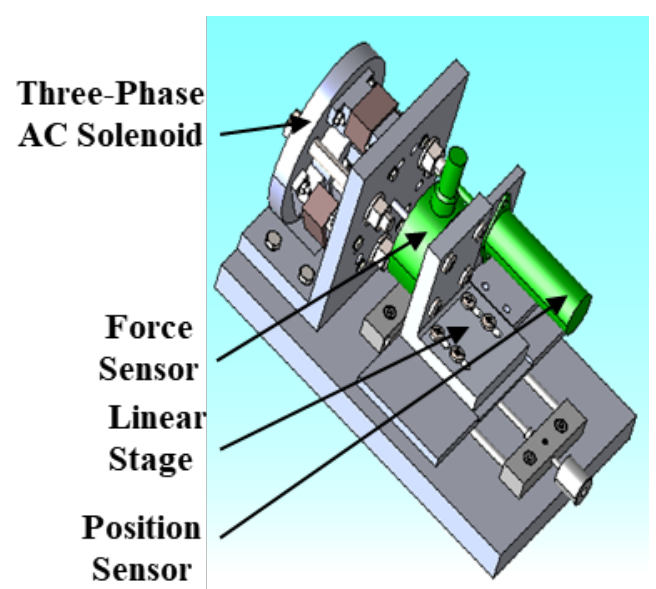

Fig. 8. Measurement apparatus for TPAC-PS prototype (Thrust force and armature position measurement)

\section{Current amplitude decrease} Current amplitude increase

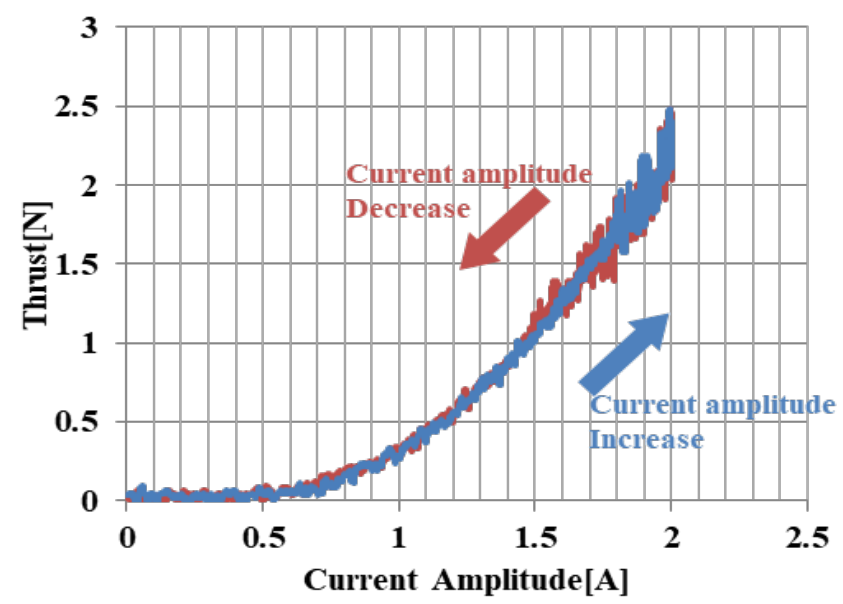

Fig. 10. Thrust force-current amplitude characteristics of TPAC-PS prototype at the fixed airgap of $0.26 \mathrm{~mm}$ at the frequency of the three-phase $\mathrm{AC}$ current of $50 \mathrm{~Hz}$

appear on the thrust force on the armature in the axial direction. Therefore, the behavior of the thrust force in the axial direction in TPAC-PS is the same as in a conventional proportional solenoid driven by the coil DC current.

\section{THREE-PHASE AC PROPORTIONAL SOLENOID PROTOTYPE}

\section{A. Three-phase AC solenoid}

Figure 8 shows the TPAC-PS prototype and its testing apparatus. It is possible to attach the force and position sensors to the linear stage, and to measure the stroke and the thrust force simultaneously. Figure 9 shows an appearance view of a prototype machine to confirm the performance of the TPAC-PS It is not installed in the triangular magnetic pole to form the

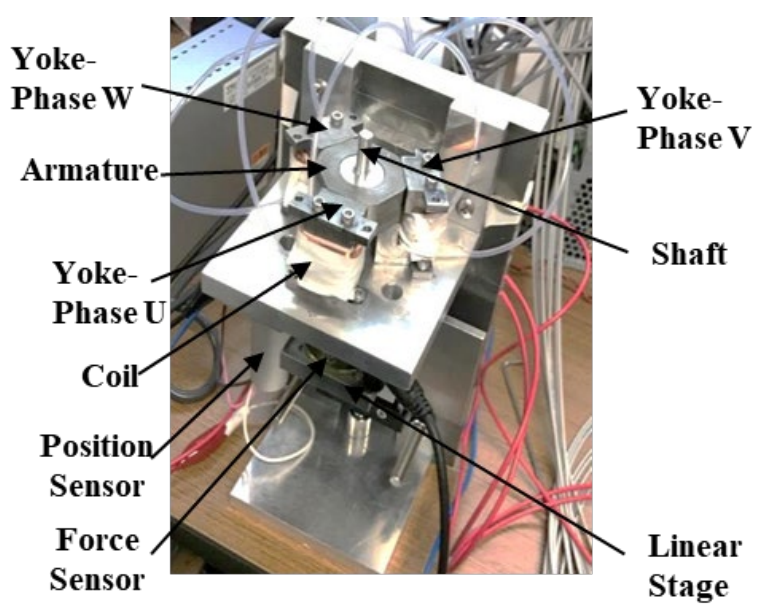

Fig. 9. Appearance view of a prototype TPAC-PS on the measurement apparatus

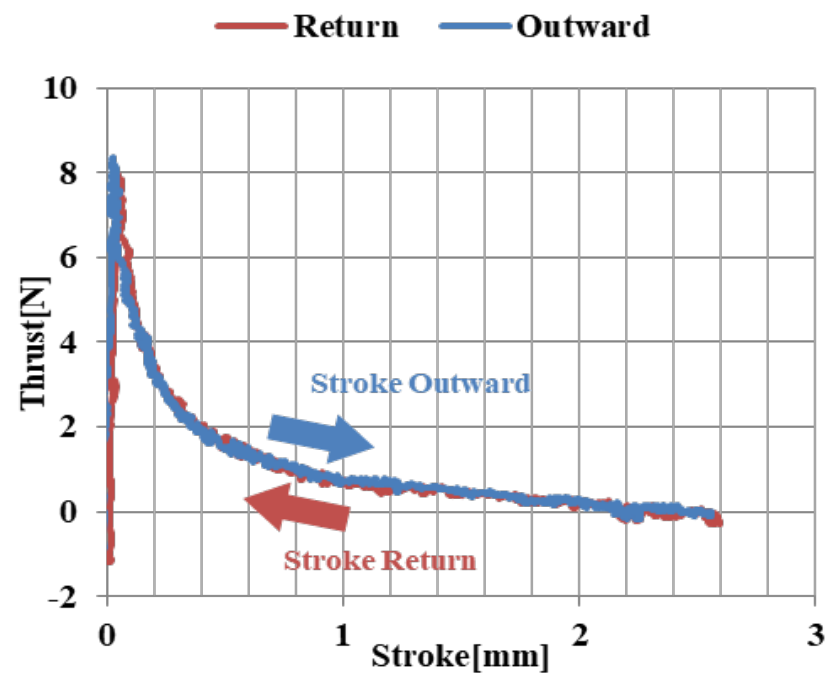

Fig. 11. Thrust force-stroke characteristics of TPAC-PS prototype at the current amplitude of $3 \mathrm{~A}$ and the frequency of the three-phase AC current of $50 \mathrm{~Hz}$

control zone on the stator, as it has been fabricated to measure the influence of the lateral force on the hysteresis in the thrust force-current characteristics.

\section{B. Measurement result of TPAC-PS prototype}

Figures 10 and 11 show the measured results of the TPACPS prototype. As this prototype does not have triangular magnetic poles on the stator, there is no control zone in those results unlike in the conventional proportional solenoid. In Fig. 10 , the current amplitude was increased or decreased from 0 to $2.0 \mathrm{~A}$ at the fixed airgap of $0.26 \mathrm{~mm}$ at the frequency of the three-phase AC current of $50 \mathrm{~Hz}$. Because the thrust force coincides with the round-trip of the current amplitude, it is effective in reducing the hysteresis. In Fig. 11, when the stroke 


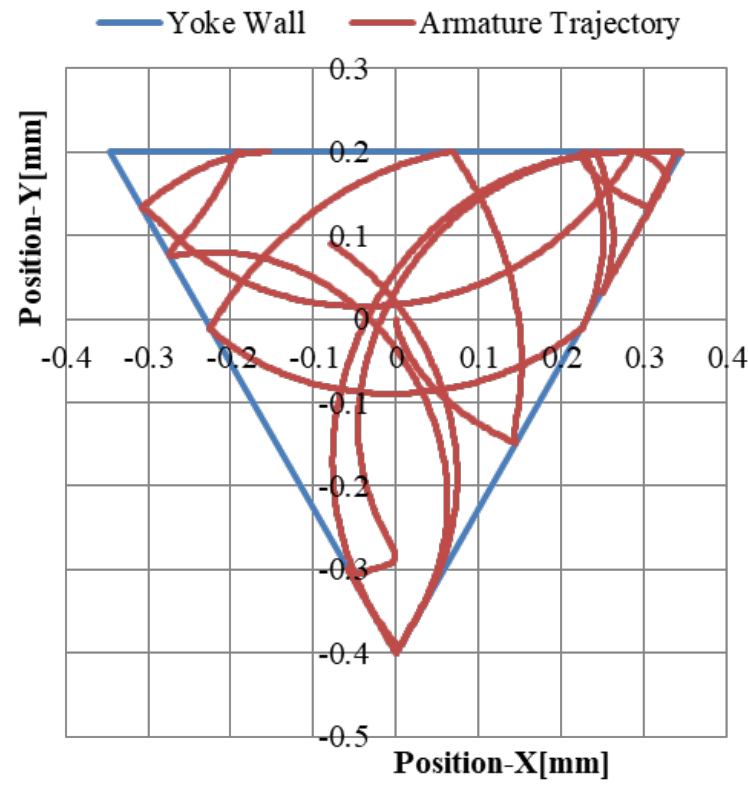

Fig. 12. Numerical analysis result for the trajectory of the center axis of the armature by lateral force from yoke

was increased or decreased at the current amplitude of $3 \mathrm{~A}$ and the frequency of the three-phase AC current of $50 \mathrm{~Hz}$, it is confirmed that the hysteresis was reduced.

\section{Reduction of hysteresis}

Although it was confirmed that thrust force hysteresis can be reduced in the TPAC-PS prototype, noise still occurred. It is conceivable that the armature is in contact with the yoke by attracting it by the lateral force. However, the theory that the hysteresis of the TPAC-PS is reduced is due to the fact that the armature is attracted to each yoke at high speed by three-phase alternating current, and the radial clearance between the armature and the yoke is always maintained. That is, the hysteresis is reduced despite the fact that the radial clearance is not maintained. Therefore, the trajectory of the armature with respect to the lateral force from each yoke was obtained by numerical analysis. The result is shown in Fig. 12. The frequency of the three-phase $\mathrm{AC}$ current is $50 \mathrm{~Hz}$, with the constant current amplitude of $3 \mathrm{~A}$ and the fixed airgap of 0.1 $\mathrm{mm}$.

From Fig. 12, contact between the armature and the yoke is also confirmed in numerical analysis. This is because the lateral force is also proportional to the square of the reciprocal of the radial clearance length due to the attractive force of the electromagnet, so the attractive force changes depending on the position. Specifically, a force is generated in the opposite direction due to the phase difference of the three-phase AC current, but the force in the opposite direction decreases by the amount attracted so far, so eventually it comes in contact with the yoke.

Figure 13 shows the time of and the reaction force on the armature contact with the yoke wall. It is observed that the contact time with the yoke is short, which is only about $10 \%$ of the total time. Moreover, since the remarkably large force in Fig. 13 is an instantaneous reaction force due to collision with the

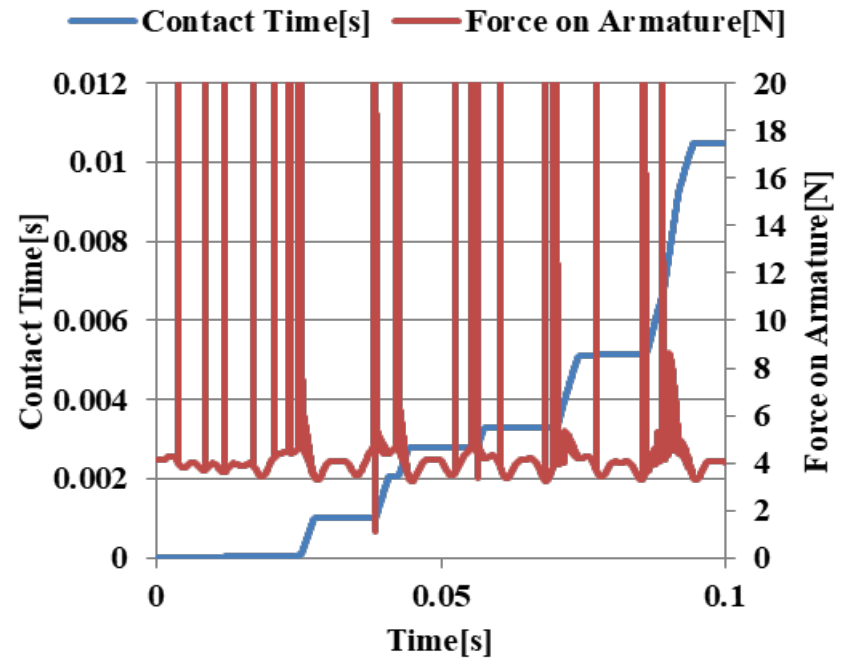

Fig. 13. Numerical analysis result for contact time and force on armature by lateral force from yoke

yoke, it is thought that the noise has almost no influence on friction. It is a similar mechanism of hysteresis reduction by dither of the conventional proportional solenoid [9]. In DC current drive like a conventional proportional solenoid, because it continues to contact the yoke, the hysteresis due to the mechanical friction also appears. On the other hand, in the TPAC-PS, it is thought that it was able to avoid being touched by lateral force switching immediately, even if it contacts the yoke.

\section{DESIGN OF TPAC-PS}

\section{A. Thrust analysis of TPAC-PS}

As it was confirmed that the TPAC-PS prototype reduces the hysteresis, it is capable of outputting a constant thrust force in proportion to the amplitude of three-phase AC current regardless of the armature position within the range of an arbitrary airgap length (control zone). Because the characteristic of a proportional solenoid is exerted by the triangular magnetic pole installed on to the stator, if a conventional proportional solenoid parts are used for the TPAC-PS, then the conventional characteristics should be reproducible [3], [10]. Figure 14 shows a TPAC-PS model that can be driven by the three-phase AC current while using a triangular pole in a conventional proportional solenoid. The stator of the TPAC-PS is trisected so that the magnetic flux loops at the magnetic pole of each UVW phase.

The thrust of the TPAC-PS was obtained by electromagnetic field analysis using the model in Fig. 14. This model has two coils for each phase yoke. One is for the DC current drive; the other is for the AC current drive. The actual TPAC-PS has one coil for each phase yoke, and the coil is driven by the $\mathrm{AC}$ current added to the bias DC current. TABLE I shows the conditions for analysis of the conventional proportional 


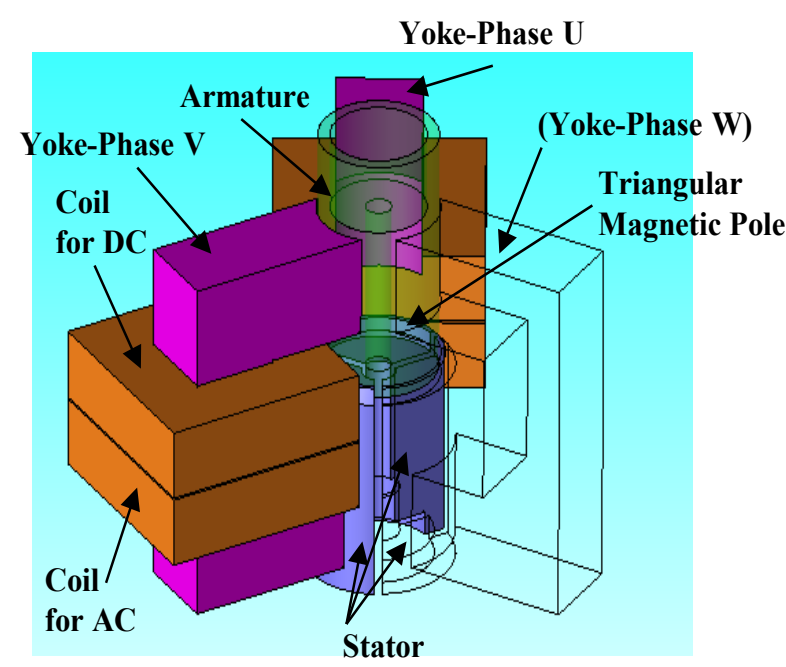

Fig. 14. TPAC-PS model

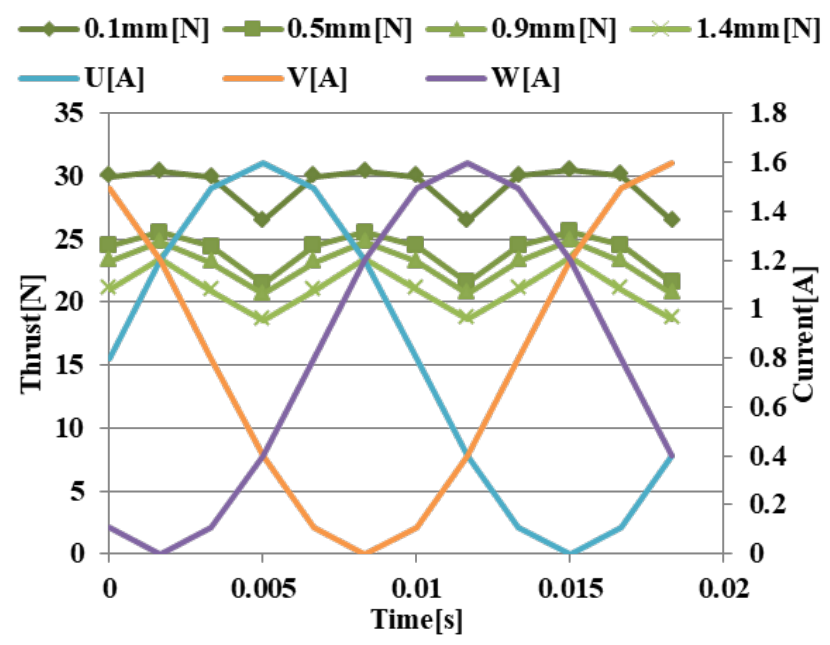

Fig. 15. Thrust force produced by the three-phase AC current of TPACPS under condition (3)

solenoid and TPAC-PS. The conditions (1), (2), and (3) are for the conventional proportional solenoid driven by the DC current, TPAC-PS driven by only the DC current, and TPAC driven by the three-phase AC current, respectively. The conditions are set so that the output of TPAC-PS was theoretically the same as the output of the conventional proportional solenoid. The result is shown in Figs. 15 and 16. The thrust force of TPAC-PS in condition (3) was averaged with the value in the slight thrust force fluctuation due to AC current in the constant airgap. In Fig. 16, TPAC-PC in the condition (2) is driven only by the DC bias current. It has the similar characteristics of the conventional proportional solenoid in condition (1). The thrust force of the TPAC-PS in condition (3) is smaller than that of the conventional proportional solenoid.
TABLE I

Electromagnetic field analysis condition

\begin{tabular}{|c|c|c|c|}
\hline & $\begin{array}{c}\text { Conventional } \\
\text { proportional }\end{array}$ & \multicolumn{2}{|c|}{ TPAC-PS } \\
\hline Condition & (1) & (2) & (3) \\
\hline Materials & $\begin{array}{l}\text { Yoke : S45C } \\
\text { Stator : S45C } \\
\text { Armature : S45C }\end{array}$ & $\leftarrow$ & $\leftarrow$ \\
\hline $\begin{array}{l}\text { Number of } \\
\text { Coil Turns [-] }\end{array}$ & 750 & $\begin{array}{l}U: 750 \\
V: 750 \\
W: 750\end{array}$ & $\leftarrow$ \\
\hline $\begin{array}{l}\text { Input Current } \\
{[\mathrm{A}]}\end{array}$ & DC $: 0.8$ & $\begin{array}{l}\text { DC : } 0.8 \\
\text { Only DC } \\
\text { current } \\
\text { drive use }\end{array}$ & $\begin{array}{l}\text { DC : } 0.8 \\
\text { AC Amplitude } \\
0.8\end{array}$ \\
\hline $\begin{array}{l}\text { AC Frequency } \\
{[\mathrm{Hz}]}\end{array}$ & - & - & 50 \\
\hline
\end{tabular}

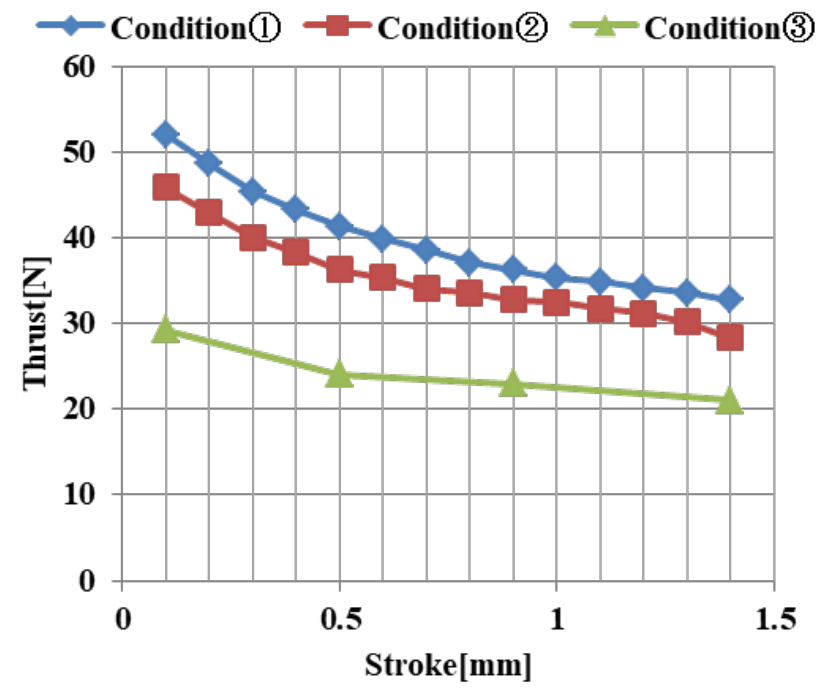

Fig. 16. Thrust force-stroke characteristics of the conventional proportional solenoid in condition (1), and TPAC-PS in conditions (2) and (3)

For the theoretical value according to Eq. (7), fluctuation on the thrust force by an $\mathrm{AC}$ component should not appear in the axial direction. However, the fluctuation is slight but did not disappear. The thrust force of TPAC-PS in condition (2) is almost the same as that of the conventional proportional solenoid in condition (1).

In the previous section, thrust force reduction and fluctuation occurred due to three-phase AC current. It is conceivable that when the AC current added on the DC bias current increases, the magnetic flux density saturates partially in the magnetic circuit of TPAC-PS. The saturation of the magnetic flux density of the material used for the TPAC-PS is represented by the B$\mathrm{H}$ curve by the electromagnetic field analysis software, as shown in Fig. 17. Figure 18 shows the thrust and the AC current of TPAC-PS when the AC current is input only to the $\mathrm{U}$ phase 


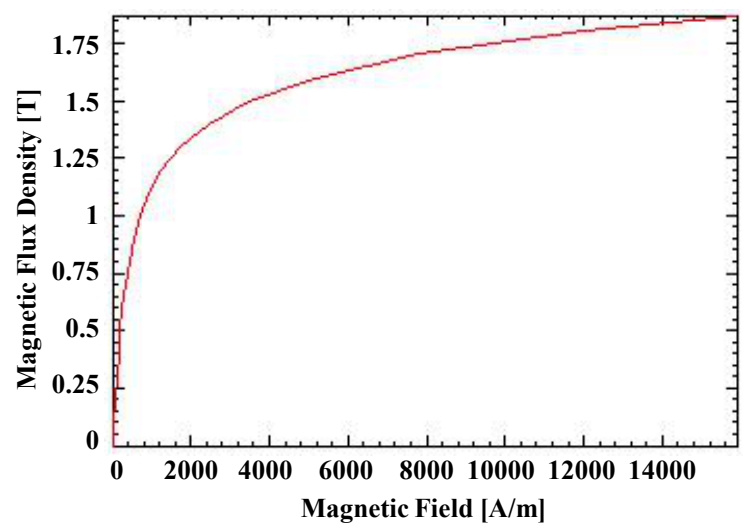

Fig. 17. B-H curve of S45C, used in electromagnetic field analysis

in the electromagnetic field analysis. Furthermore, Fig. 19 shows the thrust force of TPAC-PS driven by U-phase AC current added the DC current varying from 0.1 to $2.4 \mathrm{~A}$.

The magnetic field applied to the material is determined by the number of turns of the coil and the current value. From Fig. 18 , when the $\mathrm{AC}$ current value increases, the increase in thrust is remarkably hindered. This could explain why the thrust force of TPAC-PS is smaller the one of the conventional solenoid, and fluctuates.

\section{B. Electromagnetic field analysis under magnetic flux saturation conditions in TPAC-PS}

In the TPAC-PS model in Fig. 19 where the input current exceeds $1[\mathrm{~A}]$, the magnetic flux saturation occurs. TABLE II and Fig. 20 show the conditions for the three-phase AC current such that the AC amplitude is changed according to the DC bias current value, and the maximum value of the $\mathrm{AC}$ current added to the DC bias current is limited at $0.9[\mathrm{~A}]$.

Figures 21 and 22 show the thrust force-stroke and thrust force-current characteristics obtained by electromagnetic field analysis under the conditions shown in TABLE II. Except for the current input condition, the conventional proportional solenoid is the same as in condition (1), while the TPAC-PS is the same as in condition (3). Furthermore, the thrust force waveform driven by the three- phase $\mathrm{AC}$ current in conditions (7), is shown in Fig. 23.

From Fig. 21, the thrust of the TPAC-PS in condition (7) is

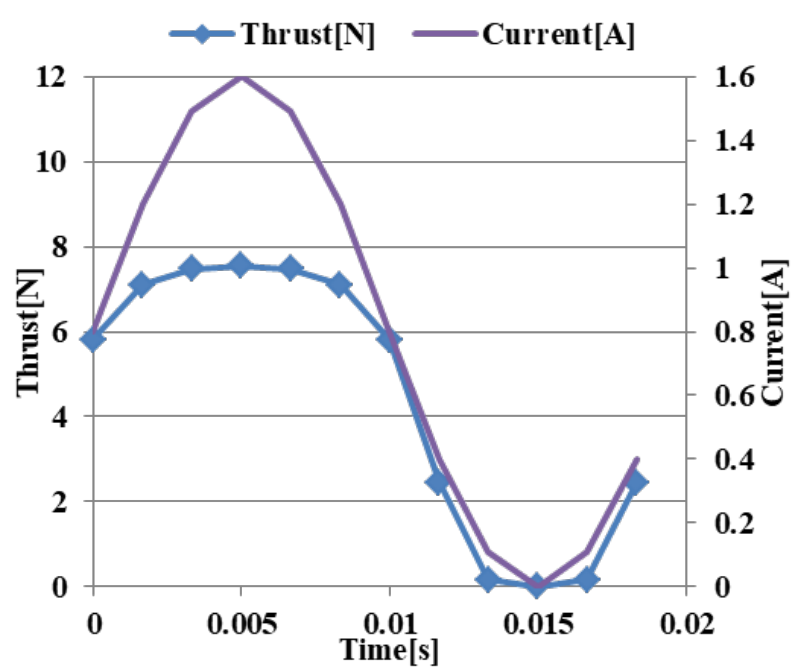

Fig. 18. Thrust force of TPAC-PS when AC current is input only to $U$ phase at the airgap of $0.1 \mathrm{~mm}$

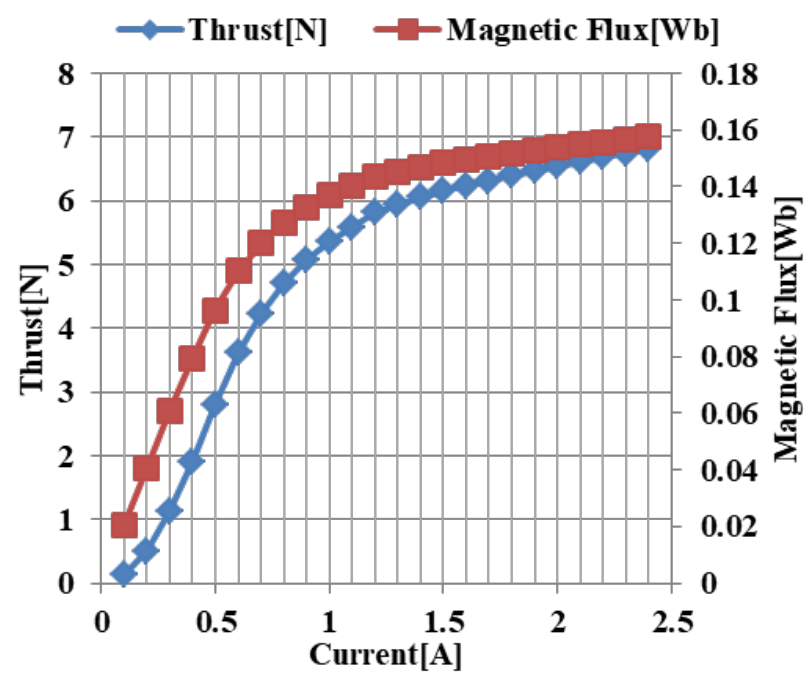

Fig. 19. Thrust force of TPAC-PS when AC current added to the DC bias current is input only to $U$ phase at the airgap of $0.1 \mathrm{~mm}$

TABLE II

Current input condition for the conventional proportional solenoid and TPAC-PS considering saturation of magnetic flux

\begin{tabular}{l|c|c|c|c|c|c|c}
\hline \hline & \multicolumn{3}{|c|}{ Conventional proportional } & \multicolumn{5}{c}{ TPAC-PS } \\
\hline Condition & 4 & 5 & $(6)$ & 7 & 8 & 9 & (10) \\
\hline DC [A] & 0.68 & 0.45 & 0.23 & 0.8 & 0.68 & 0.45 & 0.23 \\
\hline AC Amplitude [A] & - & - & - & 0.1 & 0.22 & 0.45 & 0.23 \\
\hline Maximum Current [A] & - & - & - & 0.9 & 0.9 & 0.9 & 0.46 \\
\hline \hline
\end{tabular}




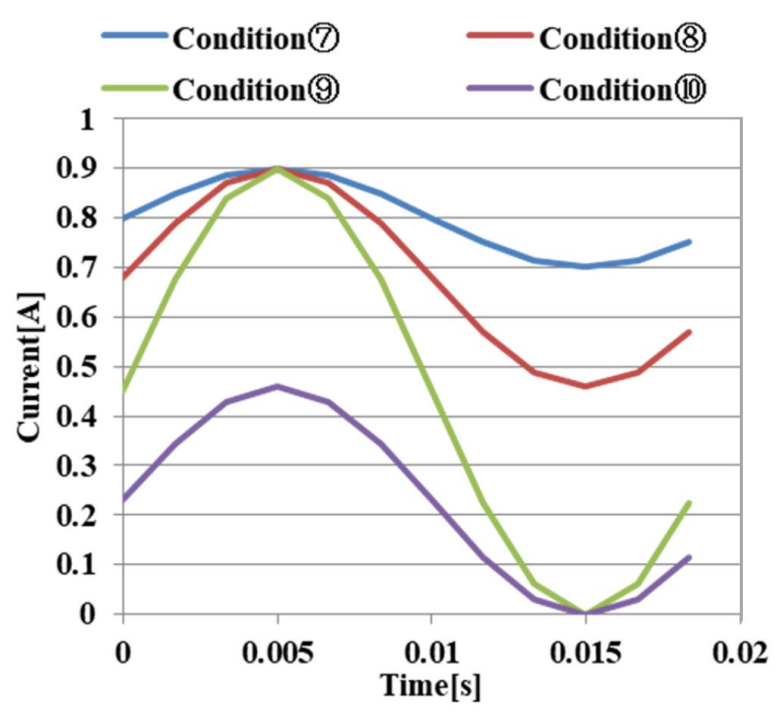

Fig. 20. Current input condition to TPAC-PS considering magnetic flux saturation (Only to U phase)
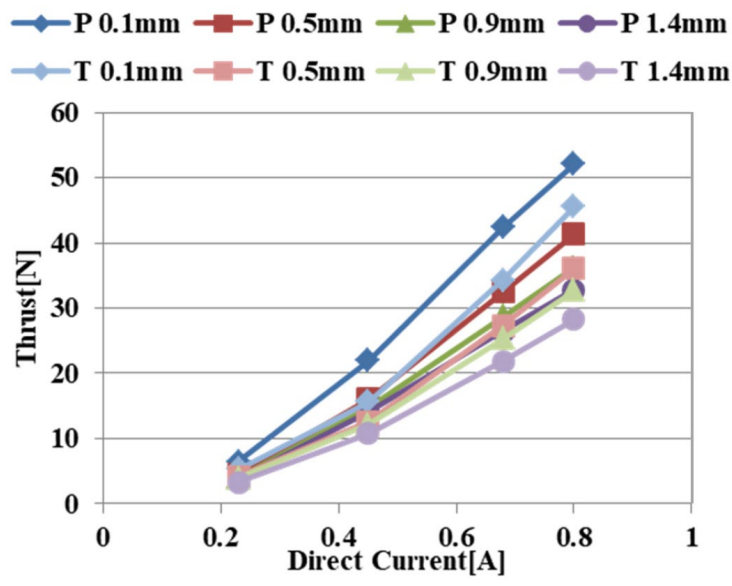

Fig. 22. Thrust force-current characteristics of the conventional proportional solenoid and TPAC-PS in various airgap length by electromagnetic field analysis

Airgap length for P: conventional proportional solenoid, and T: TPACPS

larger than that in condition (3) in Fig. 16. From Fig. 22, the thrust of the TPAC-PS is proportional to the direct current component like the conventional proportional solenoid. Moreover, from Fig. 23, the thrust fluctuation of the TPAC-PS is smaller than that in Fig. 15.

\section{CONCLUSION}

The TPAC-PS prototype reduced thrust hysteresis, due to the reduction in friction, which is realized by reducing the contact time of the armature to the sleeve by the three-phase AC current.

When TPAC-PS is manufactured with the conventional proportional solenoid parts and driven by three-phase AC current under such conditions as to avoid saturation of the

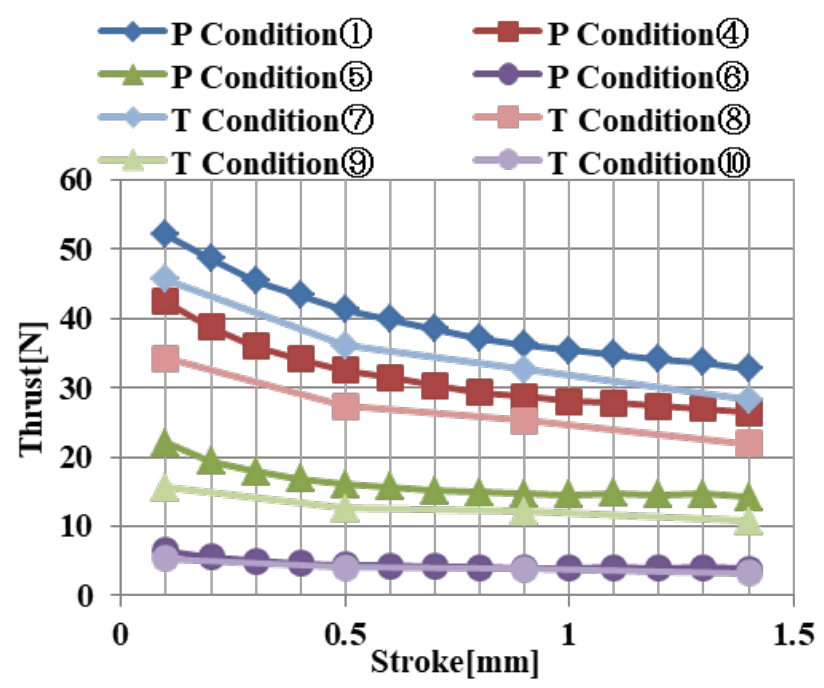

Fig. 21. Thrust force-stroke characteristics of the conventional proportional solenoid and TPAC-PS in various driving conditions by electromagnetic field analysis

Driving conditions for P: conventional proportional solenoid, and $\mathrm{T}$ : TPAC-PS

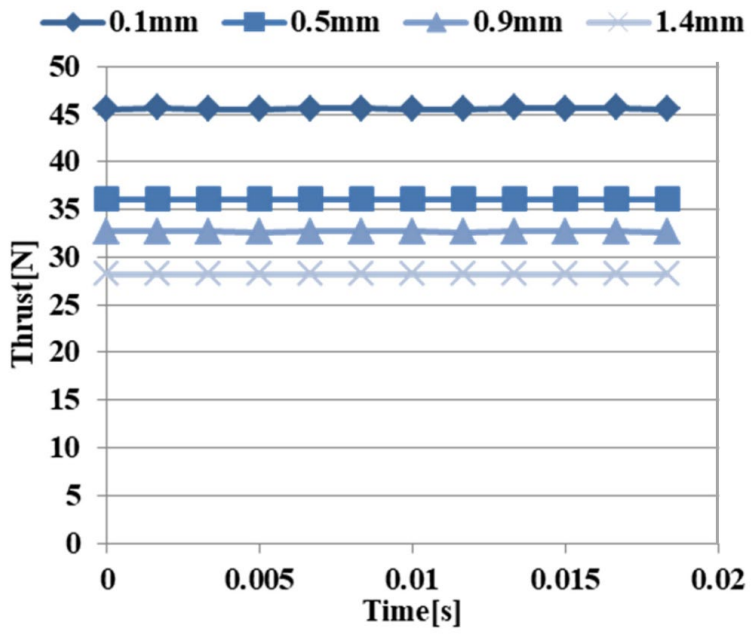

Fig. 23. Thrust force of TPAC-PS driven by the three-phase AC current under condition (7)

magnetic flux, it is confirmed by electromagnetic field analysis that its thrust force equivalent to the conventional proportional solenoid would be obtained.

\section{REFERENCES}

[1] A. Masada, K. Ebihara, "The trend and approach of linear motor and actuator in the industry," IEEJ, Vol. 109, Issue: 9, 1989, pp. 691-693.

[2] Y. Kano, K. Maeda, and A. Basak, "Analysis and reformation of characteristic of linear electromagnetic solenoid," IEEE Transactions on Magnetics, Vol. 29, Issue: 6, Nov. 1993, pp. 2929-2931.

[3] H. Yamada, S. Kihara, M. Yamaguchi, H. Nakagawa, and K. Hagiwara, "Static Thrust Improvement of a Linear PS," IEEE Translation Journal on Magnetics in Japan, Vol. 9, Issue: 6, Nov. and Dec. 1994, pp. 117-121. 
[4] H. Shokrollahi, K. Janghorban, "Soft magnetic composite materials (SMCs)," Journal of Materials Processing Technology, Vol. 189, Issues: 1-3, Jul. 2007, pp. 1-12.

[5] Y. Myung-Hwan, C. Yun-Yong, and H. Jung-Pyo, "Improvement in thrust force estimation of solenoid valve considering minor hysteresis loop," AIP Advances 7, 056607, 2017.

[6] H. Yamada, S. Kihara, M. Yamaguchi, H. Nakagawa, K. Hagiwara, and Y. Dai, "Thrust Analysis of the Linear PS for a Hydraulic Control Valve," IEEE Translation Journal on Magnetics in Japan, Vol. 9, Issue: 2, Mar. and Apr. 1994, pp. 116-123.

[7] C. Sheng-Nian, Z. Ying, P. Bao-Lin, X. Cheng-Tao, and Q. Ran, "Research on the Relationship between Drive Current Pulsating Quantity of PS Valve and Flow Hysteresis," 2012 Second International Conference on Instrumentation, Measurement, Computer, Communication and Control, 8-10 Dec. 2012.

[8] T. Hägglund, "A friction compensator for pneumatic control valves," Journal of Process Control, Vol. 12, Issue: 8, Dec. 2002, pp. 897-904.

[9] B. Armstrong-Hélouvry, P. Dupont, and C. Canudas De Wit, "A survey of models, analysis tools and compensation methods for the control of machines with friction," Automatica, Vol. 30, Issue: 7, Jul. 1994, pp.1116-1117.

[10] F. Bayat, A. F. Tehrani, and M. Danesh, "Finite element analysis of PS characteristics in hydraulic valves," International Journal of Automotive Technology, Vol. 13, Issue: 5, Aug. 2012, pp. 809-816. 